\begin{tabular}{c}
\hline INTELIGENCIA ARTIFICIAL \\
http://journal.iberamia.org/ \\
\hline
\end{tabular}

\title{
Symmetry-Based Brain Abnormality Detection Using Machine Learning
}

\author{
Mohammad A. N. Al-Azawi \\ Department of Computer Science and Management Information Systems, Oman College of Management and \\ Technology, Muscat, Oman \\ mohd.alazawi@omancollege.edu.om
}

\begin{abstract}
Medical image processing, which includes many applications such as magnetic resonance image (MRI) processing, is one of the most significant fields of computer-aided diagnostic (CAD) systems. the detection and identification of abnormalities in the magnetic resonance imaging of the brain is one of the important applications that uses magnetic resonance imaging and digital image processing techniques. In this study, we present a method that relies on the symmetry and similarity between the two lobes of the brain to determine if there are any abnormalities in the brain because tumours cause deformations in the shape of one of the lobes, which affects this symmetry. The proposed approach overcomes the challenge arising from different shapes of brain images of different people, which poses an obstacle to some approaches that rely on comparing one person's brain image with other people's brain images. In the proposed method the image of the brain is divided into two parts, one for the left lobe and the other for the right lobe. Some measures are extracted from the features of the image of each lobe separately and the distance between the corresponding metrics are calculated. These distances are used as the independent variables of the classification algorithm which determines the class to which the brain belongs. Metrics extracted from various features, such as colour and texture, were studied, discussed and used in the classification process. The proposed algorithm was applied to 366 images from standard datasets and four classifiers were tested namely Naïve Bayes (NB), random forest (RF), logistic regression (LR), and support vector machine (SVM). The obtained results from these classifiers have been discussed thoroughly where it was found that the best results were obtained from RF classifiers where the accuracy was $98.2 \%$. Finally, the results obtained and the limitations were discussed and benchmarked with state-of-the-art approaches.
\end{abstract}

Keywords: Image identification, brain MRI, Features, CAD, Brain tumour, symmetry, Classifier, AI, Machine learning.

\section{Introduction}

Magnetic resonance imaging (MRI) is one of the most well-known methods to detect and identify brain abnormality due to its availability and the high-quality images it produces [1]. The tremendous advances in computer technology over the past few decades have made Computer Aided Diagnostic (CAD) systems much more efficient and reliable. One of the important applications in CAD is magnetic resonance image (MRI) processing. These applications use image processing techniques to enhance and analyse the contents of MR images. Image enhancement techniques are used to help obtain better diagnostic results while image identification and recognition technologies are used to analyse and diagnose images.

In literature, MR image processing is divided into five stages, namely, pre-processing, feature extraction, feature selection [2], classification, and segmentation [3], [4]. The pre-processing stage includes processes such as filtering, enhancement and registration. This stage is necessary to improve the performance of the rest of the processes. In the feature extraction and selection, appropriate features are extracted and selected to be used in the 
classification process. The fourth stage is the classification which is the process of detecting and informing whether or not there is an abnormality in the brain. Finally, the segmentation process is used to isolate the abnormal region and extract some measures from it. In this research, we will focus on the feature extraction and the classification stages.

The methods of detecting and classifying brain tumours are divided into manual, semi-automatic, and fully automatic. In the first type, the user performs the entire process manually without using CAD systems, while in the second type, part of the work is done by the users and the other part is done by the CAD system. The role of the $\mathrm{CAD}$ systems is to help the user to make decisions. Finally, in fully automated, all operations are done by the computer [1], [5], [6], [7], [8]. The latter two types are the focus of the researchers as their efforts are focused on finding ways to improve the performance of automated systems.

By observing the datasets and reviewing the literature, several challenges have been identified that make anomaly detection and extraction a challenging task. The first challenge is the variation in the shape of the brain between different people and the great variety of the tumour itself in shape, size, location, and texture which make it difficult to compare one brain with another to detect any abnormalities. In addition, the shape of the brain in general and the grooves it contains made the use of digital image processing techniques more difficult [9].

In this study, the symmetry between the two lobes of the brain is used to detect any abnormality in the brain as tumours cause deformation in the shape of one of the lobes and distort this symmetry. Based on the MR images properties, the similarity around the vertical access is considered in our study. The main contribution of this research is presenting a novel approach to identify the existence of the tumours based on the symmetry between the lobes of the brain. Various features such as colour and texture have been studied and metrics extracted from these features have been discussed, studied and used in the comparison process. Four AI-based classification methods have been used and tested namely, Naïve Bayes (NB), random forest (RF), logistic regression (LR), and support vector machine (SVM).

The proposed algorithm was implemented using Python programming with JupyterLab version 2.2.6 on a PC with intel core I7 and 16 GB RAM. The algorithm was tested and applied to a dataset containing 366 various brain images constructed by selecting subsets from the following datasets:

1- "Brain MRI Images for Brain Tumor Detection" [10].

2- "Brain Tumor Classification (MRI)" [11].

The selected images are axial images with types of T1W, T2W, and Flair. The images are single-band with 256 grey levels and a size of $200 \times 200$.

The remainder of this paper is structured as follows. Section 2 presents the necessary theoretical background and reviews some of the stat-of-the-art work. The proposed approach is presented in section 3 . In section 4 , a thorough discussion of the obtained results and the limitations are presented in addition to evaluation and benchmarking with state-of-the-art approaches. Finally, the conclusions are derived in section 5.

\section{Background and Review}

Brain tumour identification has been the focus of many studies in the past few decades and as a result of the large amount of published work, many reviews are available listing the methods and techniques used. The reviews focused primarily on two approaches: non-AI-based approaches such as [1], [8], [12], [13], [14], [15], [16], [17] and artificial intelligence-based approaches such as [18], [19], [20], [21].

\subsection{AI-based and Non-AI-based approaches}

AI-based approaches utilise the principles of artificial intelligence and machine learning, such as neural nets, Fuzzy, support vector machines, decision trees, deep learning, and others, to detect and identify the abnormality region in the brain [22]. AI techniques such as classification and regression can be used in various processing stages of the detection, identification, and segmentation processes [6], [20], [23], [24].

Non-AI-based approaches use traditional image processing techniques to identify and segment the abnormality of the regions. Techniques such as thresholding, clustering, segmentation, and edge detection can be used in such kinds of approaches. They are mainly classified into pixel-based, edge-based, and region-based. Pixel-based approaches use pixel features such as the intensity, colour-band value, and location in the analysis process. Thresholding and clustering are the most commonly used pixel-based methods where the features of the pixels are used to split the image into normal and abnormal regions [25]. Region-based approaches, such as split/merge and region growing are used, these approaches deal with regions rather than pixels [26]. 


\subsection{Magnetic Resonance Imaging (MRI) of the Brain}

MRI is widely used in neurology and neurosurgery since it provides excellent details of the brain and can visualise it in three planes: axial, sagittal and coronal as shown in the example given in Figure 1 [27].

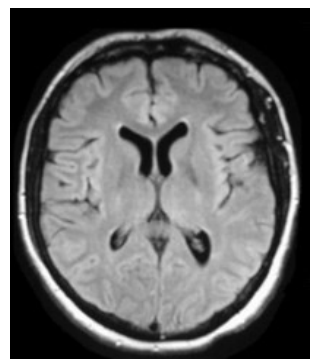

(a)

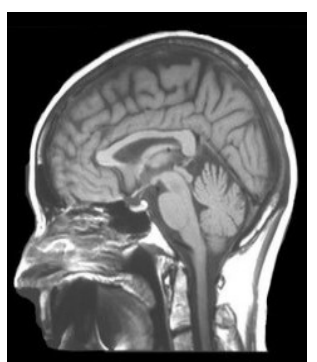

(b)

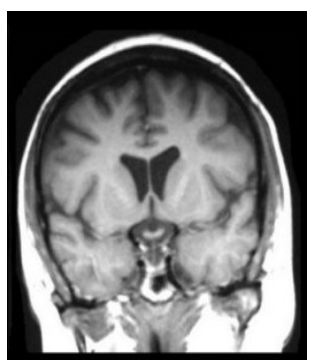

(c)

Figure 1. Brain MR images planes, (a) axial, (b) sagittal, (c) coronal [27].

Three sequences are commonly used in MRI, namely T1-weighted (T1W), T2-weighted (T2W), and Flair. T1W images are produced using short Time to Echo (TE) and short Repetition Time (TR), while T2W images are generated using longer TE and TR times. The Flair sequence is similar to a T2W but with very long TE and TR times. The three forms of sequences are shown in Figure 2 [27].

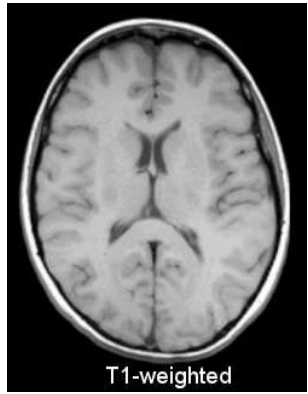

(a)

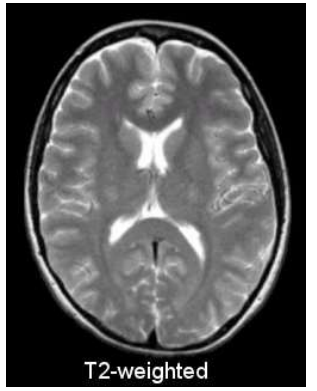

(b)

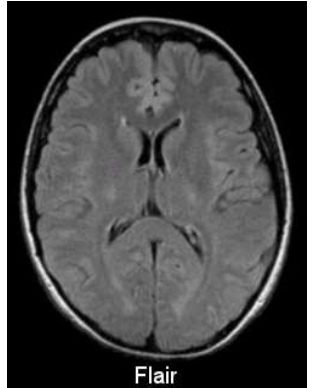

(c)

Figure 2. Three types of MRI sequences: (a) T1W, (b) T2W and (c) Flair [27].

\subsection{Pre-processing}

Pre-processing operations, such as noise removal, registration, skull stripping, intensity normalisation, and bias field correction, are important in MR image analysis as they make the images more appropriate for further processing [28]. One of the first operations is noise removal as images might be degraded due to transmission system, equipment, and lighting conditions. Several types of noise have been identified in the images such as Gaussian, Poisson, Blurred, Speckle and salt-and-pepper noise. Noise removal algorithms, such as Weiner filter, Gaussian filter, and median filter are commonly used in this stage [29]. The second important stage in pre-processing is image registration which is the process of aligning images in a dataset with one another so that it is easy to compare them with each other. Registering images needs to determine a geometric transformation that aligns one image to fit another [30].

\subsection{Related work}

In this section, we will shed light on some of the state-of-the-art related work. As was mentioned earlier many studies have been published but in our discussion, we will only consider the relevant AI-based approaches which will be used in benchmarking also.

El-Dahshan et al. suggested an approach that uses a feed-forward back-propagation artificial neural network (FP-ANN) and k-nearest neighbour (KNN) classifiers to classify whether the brain is normal or abnormal [31]. The authors applied their algorithm to 70 images from Harvard Medical School (HMS) dataset. Despite the high accuracy reported in the research, the results have a certain limitation which is the small number of images used in 
the experiments. Zöllner et al. suggested an algorithm that uses support vector machine (SVM) and applied it to 101 images from Bayer Schering Pharma dataset (BSP) and reported an accuracy of 85\% [32]. Again, the main limitation is the small number of images in the dataset. Zanaty proposed a hybrid approach, combining Fuzzy C-means (FCM), seed region growing (SRG), and Jaccard similarity coefficient (JSC) algorithm for segmentation [33]. The accuracy was reported to be $90 \%$ with no information about the dataset. In their study, Cui et al. proposed an approach that uses a localized fuzzy clustering (LFC) with spatial information for clustering the images and reported an accuracy of $83 \%$ to $95 \%$. No information was reported about the dataset or the number of images used in the experiments [34]. Sachdeva et al. used artificial neural networks (ANN) and principal components analysis artificial neural networks (PCA-ANN) to classify, segment, and extract features from MR images. The reported accuracy is $77 \%$ to $91 \%$ when applying the algorithm to a custom dataset of 55 patients [35]. Here also, the main limitation is the small number of images and the lack of variation in the images as the images were taken for the same patients.

El-Dahshan et al. used Pulse Coupled Neural Network (PCNN) for segmenting the images and applied their algorithm to a subset from the Harvard Medical School dataset (HMS) including 101 images divided into 14 normal and 87 abnormal brain images. The dataset was divided into 65 images for training and 36 for testing [36]. The authors reported an accuracy of $99 \%$ where the number of images in the test set is 36 . If 35 images were predicted correctly that means the accuracy is $97.2 \%$. The other limitation is the small number of images used in the training testing. Kumar and Vijayakumar introduced a method that used principal component analysis (PCA), radial basis function (RBF) and kernel-based SVM for segmentation and classification [37]. The authors used a subset of HMS dataset and no information is available about the number of images. The authors reported an accuracy of $94 \%$. Damodharan and Raghavan presented a neural network-based method for classification and reported an accuracy of $85 \%$ [38]. Unfortunately, there is no information available about the dataset or the number of images used in the tests. Anitha and Murugavalli suggested a method that uses K-means, self-organising map neural network (SOM$\mathrm{NN}$ ) and K-nearest neighbour (KNN), and discrete wavelet transform features for segmenting and isolating the tumour from the rest of the brain tissues. They applied their algorithm to three custom datasets including 40, 60 and 70 images respectively and reported accuracy values of $85 \%, 96.6 \%$ and $94.28 \%$ respectively. The main limitation of the results obtained from this approach is the small number of images used in the experiments [39].

Bahadure et al. used Berkeley wavelet transformation (BWT), support vector machine (SVM), adaptive neurofuzzy inference system (ANFIS), backpropagation artificial neural network (BP-ANN) and KNN in the classification process. The reported accuracy is $80.29 \%$ to $96.51 \%$ when the algorithm is applied to a custom dataset including 135 images of 15 patients [40]. Gilanie et al. used Gabor texture features and SVM to classify the images into normal and abnormal. They used three subsets from HMS dataset with 101, 75 and 70 images respectively [41]. Although the authors have reported an accuracy of $100 \%$, the small number of images used in the experiments is still a limitation. Bahadure et al. used genetic algorithm (GA) to classify and segment the tumour in brain MR images. They reported an accuracy of $92.03 \%$ using a custom dataset for 15 patients, 22 images from the Digital Imaging and Communications in Medicine (DICOM) and 44 images from the Brain Web dataset (BWD) [21]. Özyurt et al. used convolutional neural networks (CNN) with maximum fuzzy sure entropy (MFSE) and reported an accuracy of $93.1 \%$ when using CNN and SVM and an accuracy of $87.5 \%$ when using CNN and KNN. The proposed algorithm was applied to 500 samples taken from many cancer types from the Cancer Genome Atlas (TCGA) [42].

\section{Proposed Approach}

The flowchart of the proposed algorithm is shown in Figure 3, where each image in the original dataset undergoes a pre-processing process that includes some basic preparations such as noise removal and registration. Image registration, which is aligning the images with each other, is an important part of the pre-processing stage as it makes the image comparable [30]. The pre-processing stage is important to the proposed algorithm as it relies on the symmetry around the vertical axis. This stage is beyond the interest of this research as most of the available datasets include registered images and many approaches are available in the literature. The next stage is to divide the image into two images, left and right, where the metrics are extracted from the features of these images and the distance between the corresponding measures is calculated and normalised to create the dataset used in the classification process. The resulting dataset is divided into two subsets, one is used to train the model and the other is used to test the model. Finally, if the accuracy obtained is acceptable, the model can be used for prediction.

To further explain the above discussion, let $\mathbb{D}_{o}$ be the original dataset which is the set of all images $\mathcal{J}_{i}$ and given in equation (1).

$$
\mathbb{D}_{o}=\left\{\mathcal{J}_{i}: 1 \leq i \leq N\right\}
$$

where $N$ is the number of images in the dataset. 
The dataset $\mathbb{D}_{o}$ contains two types of images, normal and abnormal. If we denote the set of the normal images as $\mathbb{D}_{N}$ and the set of abnormal images as $\mathbb{D}_{A}$ then $\mathbb{D}_{o}=\mathbb{D}_{N} \cup \mathbb{D}_{A}$ and $\mathbb{D}_{N} \cap \mathbb{D}_{A}=\emptyset$. Further, we shall define the mapping $\mathcal{S}: \mathbb{D}_{o} \rightarrow \mathbb{D}_{D}$ which splits the images in $\mathbb{D}_{o}$ into tow images, left and right, i.e.:

$$
\begin{gathered}
\mathcal{S}\left(\mathcal{J}_{i}\right)=\left(L_{i}, R_{i}\right) \\
\mathbb{D}_{D}=\left\{\left(L_{i}, R_{i}\right): 1 \leq i \leq N\right\}
\end{gathered}
$$

where $\left(L_{i}, R_{i}\right)$ is an ordered pair that contains the left $L_{i}$ and the right $R_{i}$ halves of the image $\mathcal{J}_{i}$.

Each half is then undergone a metrics extraction process which can be done using the mapping $(\mathcal{F})$ given in equation (3).

$$
\begin{gathered}
\mathcal{F}(I)=M \\
M=\left\{m_{k}: 1 \leq k \leq n\right\}
\end{gathered}
$$

where $I$ is an arbitrary image, $M$ is the set of measures extracted from the features of the image and $n$ is the number of metrics extracted. The resulting set will be denoted as $\mathbb{M}$ and can be defined as $\mathbb{M}=\left\{\left(M L_{i}, M R_{i}\right): 1 \leq i \leq N\right\}$, where $M L_{i}$ and $M R_{i}$ are the metrics extracted from the left and right halves of the $i^{\text {th }}$ image respectively.

To find the distances between the left half metrics $M L_{i}$ and the right half metrics $M R_{i}$ we shall define the mapping $\mathcal{D}: \mathbb{M} \rightarrow \mathbb{d}$, where $\mathbb{d}=\left\{d_{i}: 1 \leq i \leq N\right\}$. The mapping is defined in equation (4).

$$
\begin{gathered}
\mathcal{D}\left(M L_{i}, M R_{i}\right)=d_{i} \\
d_{i}=\left\{\delta_{1}, \delta_{2}, \ldots, \delta_{n}\right\} \\
\delta_{k}=\left|m l_{k}-m r_{k}\right|
\end{gathered}
$$

where $\mathcal{D}$ is a distance measuring function, $d_{i}$ is the set of distances between the corresponding measure $\left(\delta_{k}\right)$, and $m l_{k}$ and $m r_{k}$ are the $k^{t h}$ metric of the left and right images respectively.

The resulting distances are then normalised to construct the metrics dataset $\left(\mathbb{D}_{m}\right)$ which includes all the distances between the corresponding metrics for each image and is defined by equation (5).

$$
\mathbb{D}_{m}=\left\{\left\{\overline{\delta_{1}}, \overline{\delta_{2}}, \ldots, \overline{\delta_{n}}\right\}_{1},\left\{\overline{\delta_{1}}, \overline{\delta_{2}}, \ldots, \overline{\delta_{n}}\right\}_{2}, \ldots,\left\{\overline{\delta_{1}}, \overline{\delta_{2}}, \ldots, \overline{\delta_{n}}\right\}_{N}\right\}
$$

where $\left\{\overline{\delta_{1}}, \overline{\delta_{2}}, \ldots, \overline{\delta_{n}}\right\}_{i}$ is the set of normalised distances of the $i^{\text {th }}$ image and $\overline{\delta_{k}}$ is the $k^{\text {th }}$ normalised distance. The constructed dataset to be used in the classification process $\mathbb{D}_{C}$ is given in equation (6).

$$
\mathbb{D}_{C}=\left\{\left(\left\{\overline{\delta_{1}}, \overline{\delta_{2}}, \ldots, \overline{\delta_{n}}\right\}_{1}, c_{1}\right),\left(\left\{\overline{\delta_{1}}, \overline{\delta_{2}}, \ldots, \overline{\delta_{n}}\right\}_{2}, c_{2}\right), \ldots,\left(\left\{\overline{\delta_{1}}, \overline{\delta_{2}}, \ldots, \overline{\delta_{n}}\right\}_{N}, c_{N}\right)\right\}
$$

In the above equation the ordered pair $\left(\left\{\overline{\delta_{1}}, \overline{\delta_{2}}, \ldots, \overline{\delta_{n}}\right\}_{i^{\prime}}, c_{i}\right)$ contains the set of metrics of the $i^{\text {th }}$ image and the class the image belongs to $c_{i}$. The dataset is then divided into two sets, training set $\mathbb{D}_{C}^{T r}$ and testing set $\mathbb{D}_{C}^{T s}$, the members of the sets are selected randomly.

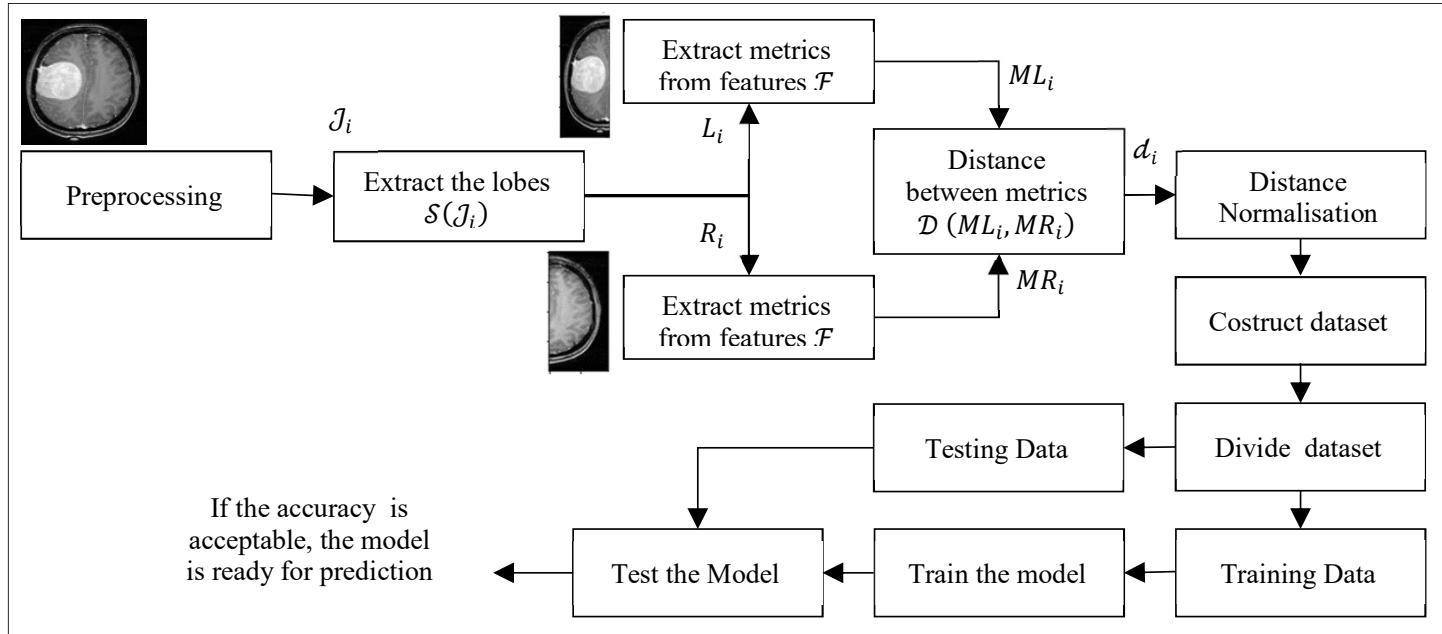

Figure 3. The proposed approach flowchart. 


\subsection{Features and metrics}

In this section, we discuss the selected features and the metrics extracted from them that are used in our approach. Two main features were considered namely, colour feature and texture features.

\section{Colour features}

Different metrics can be derived from the colour features such as statistical metrics and colour distribution metrics. In our experiments, we adopt some statistical metrics extracted from the intensity of each lobe such as mean $\left(\mu_{h}\right)$ and standard deviation $\left(\sigma_{h}\right)$ [43]. These measures can be calculated as given in equation (7).

$$
\begin{gathered}
\mu_{h}=\frac{1}{\sum_{x=0}^{255} p(x)} \sum_{x=0}^{255} x \cdot p(x) \\
\sigma_{h}=\sqrt{\frac{1}{\sum_{x=0}^{255} p(x)} \sum_{x=0}^{255}\left(x-\mu_{h}\right)^{2} \cdot p(x)}
\end{gathered}
$$

where $x$ is the grey level value and $p(x)$ is the probability of occurrence of the grey level $x$ in the image.

\section{Texture features}

Due to the nature of the brain tissues, texture features can be very useful in describing the contents of its MR images. Grey Level Co-occurrence Matrix (GLCM) is one of the important features that can be used to describe the texture of the image [44]. The metrics that were used here are Energy $\left(E_{G L C M}\right)$, Dissimilarity $\left(\delta_{G L C M}\right)$, Homogeneity $\left(h_{G L C M}\right)$, Contrast $\left(C_{G L C M}\right)$ and Correlation $\left(\rho_{G L C M}\right)$. These metrics can be calculated using the equations ( 8 through 12) given below.

$$
\begin{gathered}
E_{G L C M}=\sum_{i, j=0}^{N-1}\left(P_{i, j}\right)^{2} \\
\delta_{G L C M}=\sum_{i, j=0}^{N-1} P_{i, j} \cdot|i-j| \\
h_{G L C M}=\sum_{i, j=0}^{N-1} \frac{P_{i, j}}{1+(i-j)^{2}} \\
C_{G L C M}=\sum_{i, j=0}^{N-1} P_{i, j} \cdot(i-j)^{2} \\
\rho_{G L C M}=\sum_{i, j=0}^{N-1}\left(P_{i, j}\right) \frac{\left(i-\mu_{i}\right)\left(j-\mu_{j}\right)}{\sigma_{i} \sigma_{j}}
\end{gathered}
$$

where $i$ and $j$ are grey level values, $P_{i, j}$ is the probability of the adjacency of $i$ and $j, \mu_{i}$ and $\mu_{j}$ are the mean of the intensities of $i$ and $j$ respectively, and finally $\sigma_{i}$ and $\sigma_{j}$ are the standard deviation of the intensities $i$ and $j$ respectively.

Gabor filter is another approach that can produce important metrics which can be used to compare the tissues of the two lobes. From Gabor filters, the Energy $\left(E_{G A B}\right)$ and the Entropy $\left(\varepsilon_{G A B}\right)$ are extracted and used. The formulas and the theoretical concepts of Gabor filters can be found in references such as [41].

The third set of metrics is derived using local binary pattern (LBP) which is one of the texture spectrum descriptors. The metrics used are Entropy $\left(\varepsilon_{\varepsilon}\right)$ and Energy $\left(E_{\varepsilon}\right)$. For more information about LBP, the reader can refer to [45], [46], and [47].

\section{Results and discussion}

To simplify the presentation of the results, we shall refer to the images of the brains with abnormalities as (abnormal) and the images of the normal brains as (normal). The proposed algorithm has been applied to a dataset containing 366 images of which 158 images are for normal brains and 208 images are for brains with abnormalities. 
The metrics of the lobes of the same image is calculated and the distance between the corresponding features are extracted and normalised.

Table 1 and Figure 4 show the normalised average distances of the various metrics used in our tests. From the table and the figure, it is clear that the normalised average distances of the abnormal brains are higher than those for normal brains which proves the hypothesis that abnormalities cause deformation in one of the lobes and distort the symmetry between them.

Table 1: Normalised average distances for the metrics derived from the features of the normal and abnormal brains

\begin{tabular}{cccccccccccc}
\hline Brain & \multicolumn{10}{c}{ Normalised Average Distance } \\
\cline { 2 - 13 } Type & $\mu_{h}$ & $\sigma_{h}$ & $\varepsilon_{\varepsilon}$ & $E_{\varepsilon}$ & $E_{G L C M}$ & $\delta_{G L C M}$ & $h_{G L C M}$ & $C_{G L C M}$ & $\rho_{G L C M}$ & $E_{G A B}$ & $\varepsilon_{G A B}$ \\
\hline Normal & 0.02085 & 0.01584 & 0.00918 & 0.01172 & 0.01456 & 0.02716 & 0.02304 & 0.01830 & 0.11892 & 0.02078 & 0.02051 \\
Abnormal & 0.122003 & 0.06678 & 0.09008 & 0.10021 & 0.09087 & 0.12233 & 0.09826 & 0.12969 & 0.16648 & 0.15801 & 0.17935
\end{tabular}

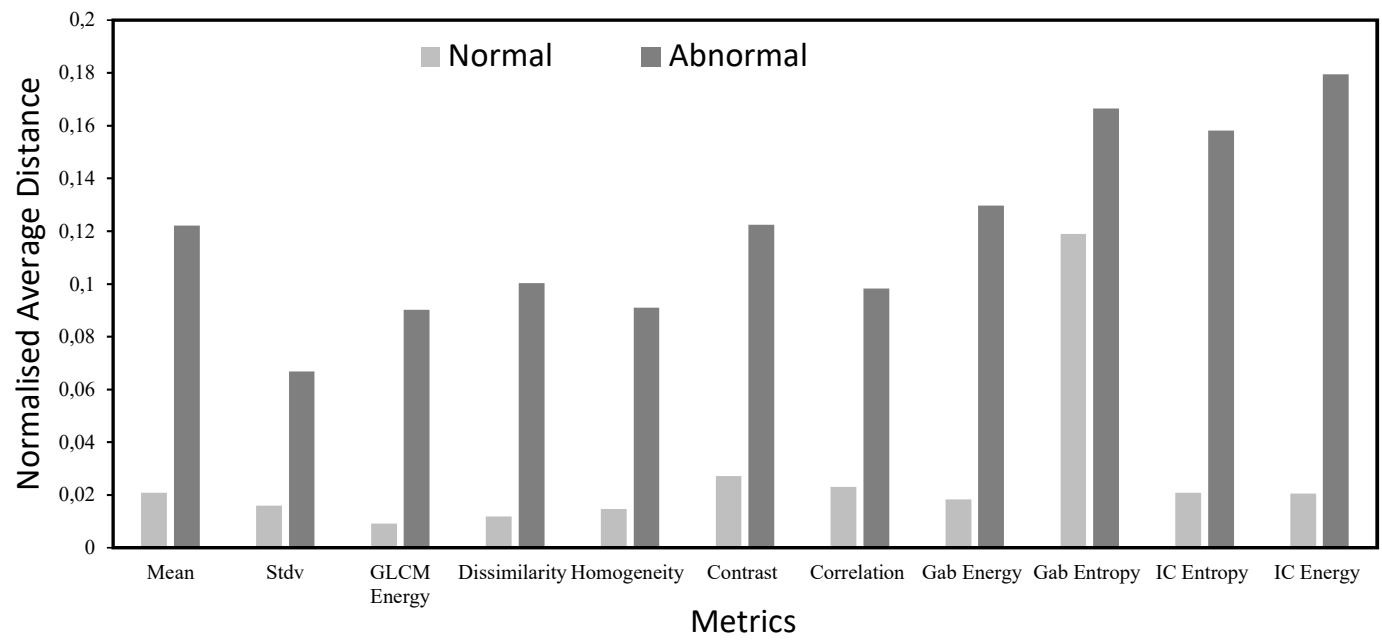

Figure 4. Normalised average distances for the metrics derived from the features of the normal and abnormal brains.

As it was mentioned earlier, four classifiers have been tested, which are Naïve Bayes (NB), random forest (RF), logistic regression (LR), and support vector machine (SVM). Figure 5 shows the learning curves of the four classifiers. In this figure, (a) shows the learning curve of the NB classifier, (b) shows the curve of the RF classifier, (c) given the learning curve of the LR, and (d) shows the curve of the SVM. From the figure, it is clear that the performance of NB is lower than others and it can be described as bad performance. The main reason for that is because NB assumes that the independent variables (the metrics) are completely independent, which is not quite accurate in our case as there is some kind of relationship and dependency among the metrics.

The best performance was obtained from RFC due to the advantages of this classifier in overcoming the overfitting and local optima problems. The second best performance is when using linear regression. Although linear regression was developed to be used with regression problems, it can be used as a classifier by adding a decision rule to it which is easy to be added. Finally, the SVM performance was intermediate and not competing because it is more efficient with problems with a high number of dimensions. 


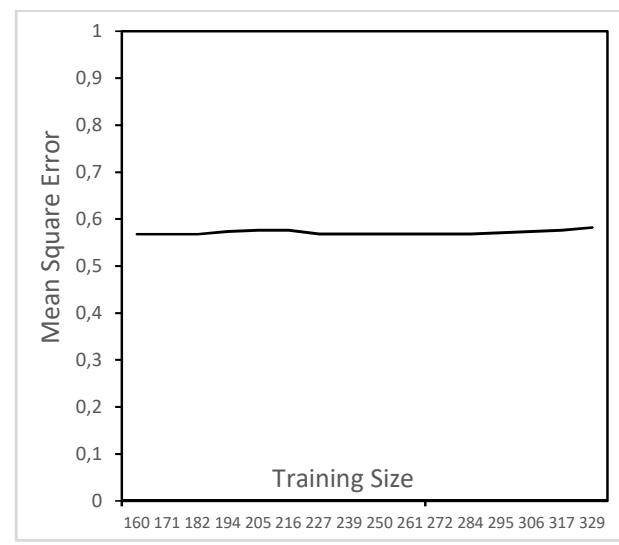

(a)

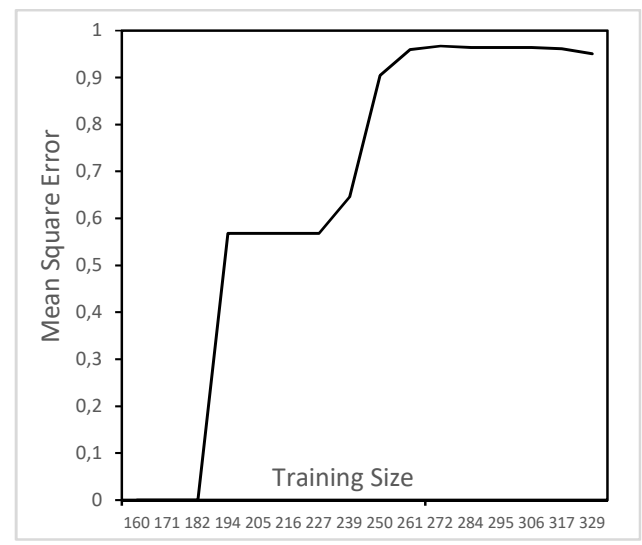

(c)

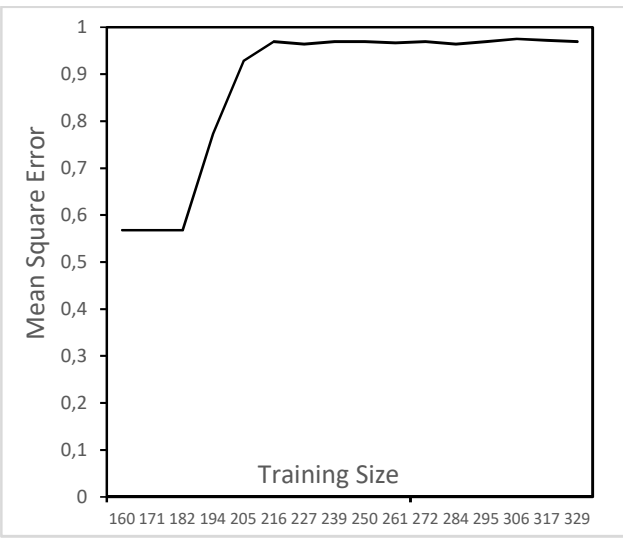

(b)

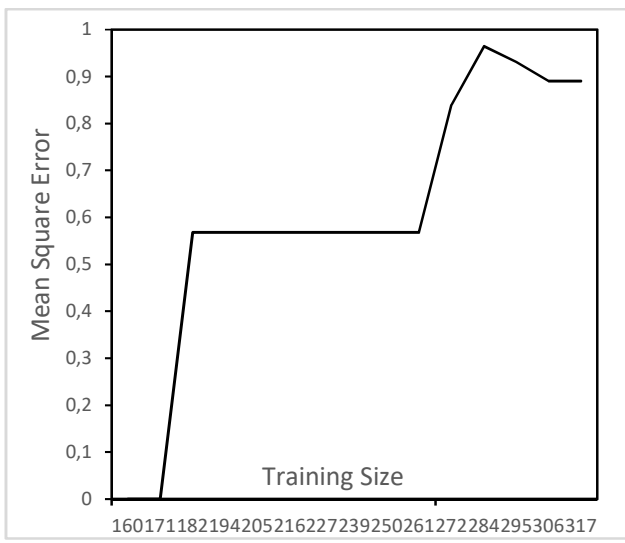

(d)

Figure 5. Learning curves for the four classifiers used in our tests (a) Naïve Bayes, (b) random forest, (c) logistic regression, and (d) support vector machine.

\subsection{Evaluation and benchmarking}

To evaluate the results obtained from the proposed algorithm, we shall adopt the precision/recall/accuracy measures which are widely used in literature. To calculate these measures, we first need to provide some definitions as follows:

Positive samples $(\mathrm{P})$ : The number of images containing abnormalities.

Negative samples $(\mathrm{N})$ : The number of images without abnormalities.

True positive (TP): The number of correctly identified images from the positive samples.

True Negative (TN): The number of correctly identified images from the negative samples.

False positive (FP): The number of incorrectly identified images from the positive samples.

False negative (FN): The number of incorrectly identified images from the negative samples.

The precision, recall, and accuracy are then can be calculated using the formulas given in equation (13).

$$
\begin{gathered}
\operatorname{Pr}=\frac{T P}{T P+F P} \\
\operatorname{Rec}=\frac{T P}{T P+F N} \\
F S=\frac{2 \times \operatorname{Pr} \times \operatorname{Rec}}{\operatorname{Pr}+\operatorname{Rec}} \\
A c c=\frac{T P+T N}{T P+T N+F P+T N}
\end{gathered}
$$

where $\operatorname{Pr}$ is the precision, $\operatorname{Rec}$ is the recall, FS is the F-Score, and Acc is the accuracy. 
Table 2 shows the results summary, from which, it is clear that the best performance is associated with RF classifier and reached $98.2 \%$ and the worst performance is associated with NB which is $59 \%$. This is due to the reason explained above. The performance of the four classifiers is shown in the confusion matrices given in Figure 6.

Table 2: Performance evaluation of the four models used

\begin{tabular}{cccccc}
\hline & Classifier & RF & LR & SVM & NB \\
\hline \multirow{5}{*}{ Positive (P) } & 63 & 63 & 63 & 63 \\
& Negative (N) & 47 & 47 & 47 & 47 \\
& True positive (TP) & 63 & 60 & 50 & 63 \\
& True negative (TN) & 45 & 47 & 47 & 2 \\
& False positive (FP) & 0 & 3 & 13 & 0 \\
& False negative (FN) & 2 & 0 & 0 & 45 \\
\hline \multirow{3}{*}{ Negative } & Precision (Pr) & 1 & 0.94 & 0.78 & 1 \\
& Recall (Rec) & 0.96 & 1 & 1 & 0.04 \\
& F-Scale (FS) & 0.98 & 0.97 & 0.88 & 0.08 \\
\hline \multirow{3}{*}{ Positive } & Precision (Pr) & 0.97 & 1 & 1 & 0.58 \\
& Recall (Rec) & 1 & 0.95 & 0.79 & 1 \\
& F-Score (FS) & 0.98 & 0.98 & 0.88 & 0.74 \\
\hline \multirow{3}{*}{ Macro Average } & Precision (Pr) & 0.98 & 0.97 & 0.89 & 0.79 \\
& Recall (Rec) & 0.98 & 0.98 & 0.9 & 0.52 \\
& F- Score (FS) & 0.98 & 0.97 & 0.88 & 0.41 \\
\hline \multirow{3}{*}{ Weighted Average } & Precision (Pr) & 0.98 & 0.97 & 0.91 & 0.76 \\
& Recall (Rec) & 0.98 & 0.97 & 0.88 & 0.59 \\
& F- Score (FS) & 0.98 & 0.97 & 0.88 & 0.46 \\
\hline & Accuracy (Acc) \% & 98.2 & 97.3 & 88.2 & 59 \\
& Training time (ms) & 777 & 407 & 382 & 392 \\
\hline
\end{tabular}

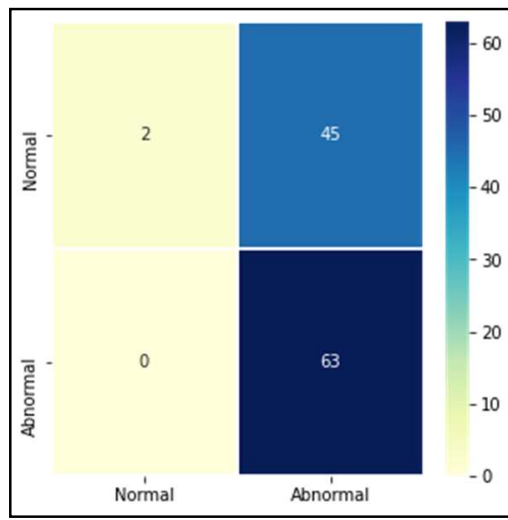

(a)

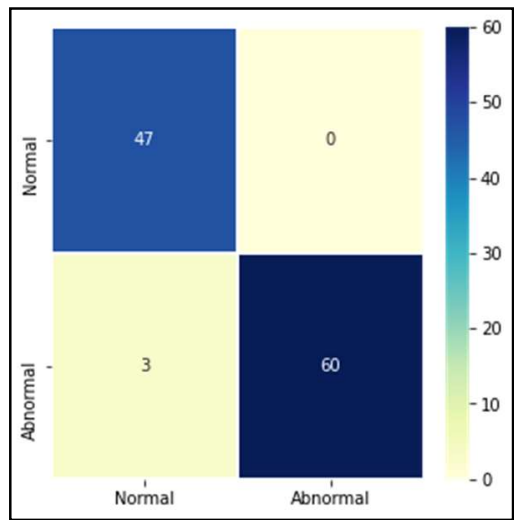

(c)

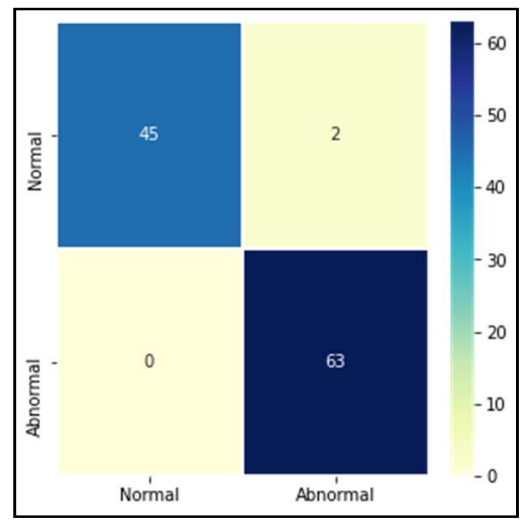

(b)

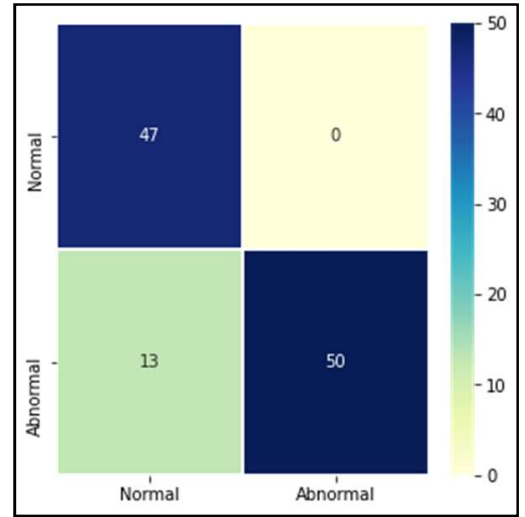

(d)

Figure 6. Confusion metrics of (a) Naïve Bayes, (b) random forest, (c) logistic regression, and (d) support vector machine. 
To benchmark the proposed approach, we have selected some state-of-the-art methods, which are presented in the following discussion. Unfortunately, there is no single method to evaluate the performance of the classification approaches, but from the literature, we found that the most common evaluation method is the accuracy. In our benchmarking, we considered mainly the accuracy and the number of images used in the dataset. The methods that have been used in the benchmarking are listed below in Table 3. From the table, it is clear that the proposed algorithm has given very good accuracy values with RF and LR. Although some methods have reported 100\% accuracy such as GIL [41], there is a certain limitation with this algorithm which is the small number of images used in testing.

Table 3: Benchmarking with state-of-the-art methods

\begin{tabular}{|c|c|c|c|c|c|}
\hline \# & Research & Approach & Dataset & Number of images & Accuracy \% \\
\hline 1. & $\overline{E L D}[31]$ & PCA, KNN & HMS & 70,10 normal and 60 abnormal & 98 \\
\hline 2. & $\mathrm{ZO}[32]$ & SVM & BSP & 101 & 85 \\
\hline 3. & $\mathrm{ZN}[33]$ & FMC, SRG, JSC & - & - & 90 \\
\hline 4. & CUI [34] & LFC & - & - & 83 to 95 \\
\hline 5. & $\mathrm{SAC}[35]$ & ANN PCA-ANN & Custom & 55 patients & 77 to 91 \\
\hline 6. & ELD [36] & PCNN & HMS & 101,14 normal and 87 abnormal & 99 \\
\hline 7. & KV [37] & PCA, RBF, SVM & HMS & - & 94 \\
\hline 8. & DR [38] & ANN & - & - & 85 \\
\hline \multirow[t]{3}{*}{9.} & AM [39] & K-means, SOM-NN, KNN & Custom & 40 & 85 \\
\hline & & & Custom & 60 & 96.6 \\
\hline & & & Custom & 70 & 94.28 \\
\hline 10. & BAH-1 [40] & BWT, SVM, ANFIS, BP-ANN, KNN & Custom & 135 images of 15 patients & 80.29 to 96.51 \\
\hline \multirow[t]{3}{*}{11.} & GIL [41] & Gabor, SVM & HMS & 101,14 normal/ 87 abnormal & 100 \\
\hline & & & HMS & 75,15 normal $/ 60$ abnormal & 100 \\
\hline & & & HMS & 70,10 normal $/ 60$ abnormal & 100 \\
\hline \multirow[t]{3}{*}{12.} & BAH-2 [21] & GA & Custom & 15 patients & 92.03 \\
\hline & & & DICOM & 22 & \\
\hline & & & BWD & 44 & \\
\hline \multirow[t]{2}{*}{13.} & $\mathrm{OZ}$ [42] & CNN and SVM & TCGA & 500 & 93.1 \\
\hline & & $\mathrm{CNN}$ and $\mathrm{KNN}$ & & & 87.5 \\
\hline \multirow[t]{3}{*}{14.} & Proposed & Symmetry, & & & \\
\hline & & LR & & 366 & 97.3 \\
\hline & & $\mathrm{RFC}$ & & 366 & 98.2 \\
\hline
\end{tabular}

\subsection{Limitations}

By studying the wrongly classified brain images we found that the main limitation is when the tumour itself is symmetrical around the vertical axis, which is very rare, as shown in Figure 7 (a) or when the effect of other parts such as the eyeballs is more than the tumour itself as shown in Figure 7 (b). The lack of symmetry around the vertical axis, Figure 7 (c), and the noise produced during the imaging process Figure 7 (d) may also affect the accuracy of the result obtained from the algorithm. Except for the first limitation, the effect of the other three limitations can be reduced in the preprocessing stage by selecting efficient techniques for filtering, registration, and skull stripping.

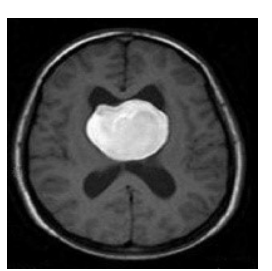

(a)

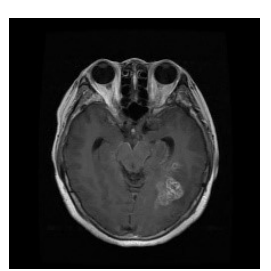

(b)

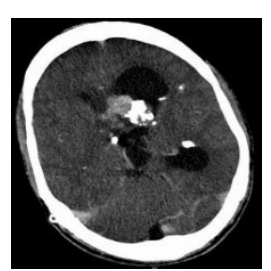

(c)

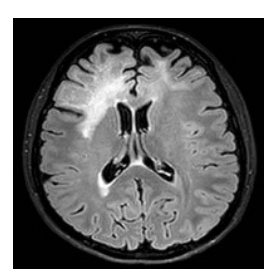

(d)

Figure 7. Samples of the wrongly identified images, (a) symmetrical tumour, (b) the effect of skull and eyeballs, (c) badly registered image, (d) the presence of noise and distortion in imaging process

\section{Conclusions}

In this study, we presented a novel approach for detecting abnormalities or tumours in the brain by processing its MR image. The proposed used AI-based classifiers to classify the brain images into normal and abnormal. The independent variables are derived from the image of the brain by dividing the image into two images one contains the left lobe and the other contains the right lobe. The distance between metrics was considered as an independent variable. Since we selected eleven metrics derived from various features of the image, the number of independent variables used in the training process was eleven. Four classifiers were tested and evaluated namely, NB, SVM, LR, 
and RF. The best performance was associated with the RF classifier where the accuracy reached $98.2 \%$ when applied to the dataset which contains 366 images.

The results of the proposed algorithm were evaluated using precision/recall, F-score, accuracy and confusion matrix and benchmarked with state-of-the-art methods considering both the accuracy and the number of images in the dataset. The benchmarking showed that the proposed method has produced very satisfactory results.

Finally, the limitation of the proposed method was discussed, and reasons were identified. The main reasons that affect the accuracy are due to poor pre-processing.

Further work can be done in the future considering testing more features and applying the proposed algorithm to other body organs which have symmetry such as lungs and breasts.

\section{References}

[1] M. S. H. Al-Tamimi and G. Sulong, "Tumor brain detection through MR images: A review of literature," J. Theor. Appl. Inf. Technol., vol. 62, no. 2, pp. 387-403, 2014.

[2] M. A. N. Al-Azawi, "Mutual Information-Based Optimum Metrics Identification in Symmetry-Based Brain Abnormality Detection," J. Phys. Conf. Ser., vol. 1998, no. 1, 2021, doi: 10.1088/17426596/1998/1/012012.

[3] M. Al-Azawi, "Saliency-Based Brain Abnormality Identification in Magnetic Resonance Images," Sci. Vis., vol. 13, no. 2, pp. 24-49, 2021, doi: 10.26583/sv.13.2.03.

[4] D. M. Kumar, D. Satyanarayana, and M. N. G. Prasad, "An improved Gabor wavelet transform and rough K-means clustering algorithm for MRI brain tumor image segmentation," Multimed. Tools Appl., Oct. 2020, doi: 10.1007/s11042-020-09635-6.

[5] Q. Li, Z. Yu, Y. Wang, and H. Zheng, "Tumorgan: A multi-modal data augmentation framework for brain tumor segmentation," Sensors (Switzerland), vol. 20, no. 15. pp. 1-16, 2020, doi: 10.3390/s20154203.

[6] G. Tomasila and A. W. Rahardjo Emanuel, "MRI image processing method on brain tumors: A review," in AIP Conference Proceedings, 2020, vol. 2296, p. 20023, doi: 10.1063/5.0030978.

[7] H. Peni Agustin Tjahyaningtijas, "Brain Tumor Image Segmentation in MRI Image," IOP Conf. Ser. Mater. Sci. Eng., vol. 336, no. 1, 2018, doi: 10.1088/1757-899X/336/1/012012.

[8] S. Saritha and N. Amutha Prabha, "A comprehensive review: Segmentation of MRI images—brain tumor," Int. J. Imaging Syst. Technol., vol. 26, no. 4, pp. 295-304, 2016, doi: 10.1002/ima.22201.

[9] G. Wang, W. Li, S. Ourselin, and T. Vercauteren, “Automatic brain tumor segmentation using convolutional neural networks with test-time augmentation," in Lecture Notes in Computer Science (including subseries Lecture Notes in Artificial Intelligence and Lecture Notes in Bioinformatics), 2019, vol. 11384 LNCS, pp. 61-72, doi: 10.1007/978-3-030-11726-9_6.

[10] N. Chakrabarty, "Brain MRI Images for Brain Tumor Detection," 2018. https://www.kaggle.com/navoneel/brain-mri-images-for-brain-tumor-detection (accessed Oct. 01, 2020).

[11] Sartaj, "Brain Tumor Classification (MRI)," 2020. https:/www.kaggle.com/sartajbhuvaji/brain-tumorclassification-mri (accessed Aug. 01, 2020).

[12] P. N. Kale and R. T. Vyavahare, "MRI Brain Tumor Segmentation Methods- A Review," 1271| Int. J. Curr. Eng. Technol., vol. 6, no. 4, pp. 1271-1280, 2016, Accessed: Dec. 01, 2020. [Online]. Available: http://inpressco.com/category/ijcet.

[13] S. R. Rajput and M. S. Raval, "A Review on End-To-End Methods for Brain Tumor Segmentation and Overall Survival Prediction," arXiv, 2020, doi: 10.32010/26166127.

[14] S. M. Kulkarni and G. Sundari, "A Review on Image Segmentation for Brain Tumor Detection," in Proceedings of the 2nd International Conference on Electronics, Communication and Aerospace Technology, ICECA 2018, 2018, vol. 3, pp. 552-555, doi: 10.1109/ICECA.2018.8474893.

[15] A. Wadhwa, A. Bhardwaj, and V. Singh Verma, "A review on brain tumor segmentation of MRI images," Magnetic Resonance Imaging, vol. 61. pp. 247-259, 2019, doi: 10.1016/j.mri.2019.05.043.

[16] A. Anand and H. Kaur, "Survey on Segmentation of Brain Tumor: A Review of Literature," Ijarcce, vol. 5, no. 1, pp. 79-82, 2016, doi: 10.17148/ijarcce.2016.5118.

[17] S. Tripathi, R. S. Anand, and E. Fernandez, “A Review of Brain MR Image Segmentation Techniques," Int. J. Res. Anal. Rev., vol. 5, no. 2, p. 1295, 2018, [Online]. Available: http://ijrar.com/.

[18] A. Işin, C. Direkoğlu, and M. Şah, "Review of MRI-based Brain Tumor Image Segmentation Using Deep Learning Methods," in Procedia Computer Science, 2016, vol. 102, pp. 317-324, doi: 10.1016/j.procs.2016.09.407.

[19] S. M. Anwar, M. Majid, A. Qayyum, M. Awais, M. Alnowami, and M. K. Khan, "Medical Image Analysis 
using Convolutional Neural Networks: A Review," J. Med. Syst., vol. 42, no. 11, 2018, doi: 10.1007/s10916-018-1088-1.

[20] L. Pei, L. Vidyaratne, M. M. Rahman, and K. M. Iftekharuddin, "Context aware deep learning for brain tumor segmentation, subtype classification, and survival prediction using radiology images," Sci. Rep., vol. 10, no. 1, pp. 1-11, 2020, doi: 10.1038/s41598-020-74419-9.

[21] N. B. Bahadure, A. K. Ray, and H. P. Thethi, "Comparative Approach of MRI-Based Brain Tumor Segmentation and Classification Using Genetic Algorithm," J. Digit. Imaging, vol. 31, no. 4, pp. 477-489, Aug. 2018, doi: 10.1007/s10278-018-0050-6.

[22] J. Chen, C. Yang, G. Xu, and L. Ning, "Image Segmentation Method Using Fuzzy C Mean Clustering Based on Multi-Objective Optimization," J. Phys. Conf. Ser., vol. 1004, no. 1, p. 012035, Apr. 2018, doi: 10.1088/1742-6596/1004/1/012035.

[23] W. Wu et al., "An Intelligent Diagnosis Method of Brain MRI Tumor Segmentation Using Deep Convolutional Neural Network and SVM Algorithm," Comput. Math. Methods Med., vol. 2020, 2020, doi: $10.1155 / 2020 / 6789306$.

[24] C. C. Benson, V. Deepa, V. L. Lajish, and K. Rajamani, "Brain tumor segmentation from MR brain images using improved fuzzy c-means clustering and watershed algorithm," in 2016 International Conference on Advances in Computing, Communications and Informatics, ICACCI 2016, Nov. 2016, pp. 187-192, doi: 10.1109/ICACCI.2016.7732045.

[25] M. A. N. Al-azawi, "Image Thresholding using Histogram Fuzzy Approximation," Int. J. Comput. Appl., vol. 83, no. 9, pp. 36-40, 2013, doi: 10.5120/14480-2781.

[26] I. Zabir, S. Paul, M. A. Rayhan, T. Sarker, S. A. Fattah, and C. Shahnaz, "Automatic brain tumor detection and segmentation from multi-modal MRI images based on region growing and level set evolution," in 2015 IEEE International WIE Conference on Electrical and Computer Engineering, WIECON-ECE 2015, Mar. 2016, pp. 503-506, doi: 10.1109/WIECON-ECE.2015.7443979.

[27] E. S. H. Ibrahim and R. E. Gabr, "MRI basics," in Heart Mechanics: Magnetic Resonance ImagingMathematical Modeling, Pulse Sequences, and Image Analysis, 2017, pp. 81-120.

[28] Z. Akkus, A. Galimzianova, A. Hoogi, D. L. Rubin, and B. J. Erickson, "Deep Learning for Brain MRI Segmentation: State of the Art and Future Directions," Journal of Digital Imaging, vol. 30, no. 4. Springer New York LLC, pp. 449-459, Aug. 01, 2017, doi: 10.1007/s10278-017-9983-4.

[29] N. Kumar and M. Nachamai, "Noise Removal and Filtering Techniques used in Medical Images," Orient. J. Comput. Sci. Technol., vol. 10, no. 1, pp. 103-113, Mar. 2017, doi: 10.13005/ojcst/10.01.14.

[30] P. J. Kostelec and S. Periaswamy, "Image Registration for MRI," Mod. Signal Process., vol. 46, pp. 161184, 2003.

[31] E. S. A. El-Dahshan, T. Hosny, and A. B. M. Salem, "Hybrid intelligent techniques for MRI brain images classification," Digit. Signal Process. A Rev. J., vol. 20, no. 2, pp. 433-441, 2010, doi: 10.1016/j.dsp.2009.07.002.

[32] F. G. Zöllner, K. E. Emblem, and L. R. Schad, "SVM-based glioma grading: Optimization by feature reduction analysis," Z. Med. Phys., vol. 22, no. 3, pp. 205-214, Sep. 2012, doi: 10.1016/j.zemedi.2012.03.007.

[33] EA Zanaty, "Determination of Gray Matter (GM) and White Matter (WM) Volume in Brain Magnetic Resonance Images (MRI)," Int. J. Comput. Appl., vol. 45, no. 3, pp. 16-22, 2012, [Online]. Available: https://pdfs.semanticscholar.org/1757/d8a0e6292835eb598bc9c40fac7b71029822.pdf.

[34] W. Cui, Y. Wang, Y. Fan, Y. Feng, and T. Lei, "Localized FCM clustering with spatial information for medical image segmentation and bias field estimation," Int. J. Biomed. Imaging, vol. 2013, 2013, doi: 10.1155/2013/930301.

[35] J. Sachdeva, V. Kumar, I. Gupta, N. Khandelwal, and C. K. Ahuja, "Segmentation, feature extraction, and multiclass brain tumor classification," J. Digit. Imaging, vol. 26, no. 6, pp. 1141-1150, 2013, doi: 10.1007/s10278-013-9600-0.

[36] E. A. S. El-Dahshan, H. M. Mohsen, K. Revett, and A. B. M. Salem, "Computer-aided diagnosis of human brain tumor through MRI: A survey and a new algorithm," Expert Syst. Appl., vol. 41, no. 11, pp. 55265545, 2014, doi: 10.1016/j.eswa.2014.01.021.

[37] P. Kumar and B. Vijayakumar, "Brain Tumour Mr Image Segmentation and Classification Using by PCA and RBF Kernel Based Support Vector Machine," Middle-East J. Sci. Res., vol. 23, no. 9, pp. 2106-2116, 2015, doi: 10.5829/idosi.mejsr.2015.23.09.22458.

[38] S. Damodharan and D. Raghavan, "Combining tissue segmentation and neural network for brain tumor detection," Int. Arab J. Inf. Technol., vol. 12, no. 1, pp. 42-52, 2015.

[39] V. Anitha and S. Murugavalli, "Brain tumour classification using two-tier classifier with adaptive 
segmentation technique," IET Comput. Vis., vol. 10, no. 1, pp. 9-17, 2016, doi: 10.1049/iet-cvi.2014.0193.

[40] N. B. Bahadure, A. K. Ray, and H. P. Thethi, "Image Analysis for MRI Based Brain Tumor Detection and Feature Extraction Using Biologically Inspired BWT and SVM,” Int. J. Biomed. Imaging, vol. 2017, 2017, doi: $10.1155 / 2017 / 9749108$.

[41] G. Gilanie, U. I. Bajwa, M. M. Waraich, Z. Habib, H. Ullah, and M. Nasir, "Classification of normal and abnormal brain MRI slices using Gabor texture and support vector machines," Signal, Image Video Process., vol. 12, no. 3, pp. 479-487, 2018, doi: 10.1007/s11760-017-1182-8.

[42] F. Özyurt, E. Sert, E. Avci, and E. Dogantekin, "Brain tumor detection based on Convolutional Neural Network with neutrosophic expert maximum fuzzy sure entropy,” Meas. J. Int. Meas. Confed., vol. 147, 2019, doi: 10.1016/j.measurement.2019.07.058.

[43] M. Al-Azawi, "Saliency-Based Image Retrieval using Colour Histogram Feature," IEEE Int. Conf. Electr. Electron. Comput. Mech. Comput. EECCMC, 2018.

[44] M. Al-Azawi, "Computational Intelligence-based Semantic Image Background Identification using ColourTexture Feature," Int. J. Comput. Appl., vol. 180, no. 10, pp. 27-31, Jan. 2018, doi: $10.5120 /$ ijca2018916165.

[45] M. S. Nixon and A. S. Aguado, "Introduction to texture description, segmentation, and classification," Featur. Extr. Image Process. Comput. Vis., pp. 399-434, 2012, doi: 10.1016/B978-0-12-396549-3.000082.

[46] Z. Camlica, H. R. Tizhoosh, and F. Khalvati, "Medical Image Classification via SVM Using LBP Features from Saliency-Based Folded Data," in 2015 IEEE 14th International Conference on Machine Learning and Applications (ICMLA), Dec. 2015, pp. 128-132, doi: 10.1109/ICMLA.2015.131.

[47] S. Abbasi and F. Tajeripour, "Detection of brain tumor in 3D MRI images using local binary patterns and histogram orientation gradient," Neurocomputing, vol. 219, pp. 526-535, 2017, doi: 10.1016/j.neucom.2016.09.051. 